Artigo

\title{
Estudo cromossômico no sangue periférico de pacientes com diferentes tipos de leucemia do Hospital de Base, São José do Rio Preto - SP
}

\author{
Agnes C. Fett-Conte \\ Cristina B. Vendrame-Goloni ${ }^{2}$ \\ Carlos M. Homsi ${ }^{3}$ \\ Leila N.B. Borim ${ }^{4}$ \\ Paulo A. Zola ${ }^{3}$ \\ Octávio Ricci ${ }^{3}$
}

\begin{abstract}
A análise das alterações cromossômicas em leucemias tem uma aplicação direta no diagnóstico, prognóstico e tratamento dos pacientes. Além disso, permite o entendimento dos processos biológicos envolvidos na carcinogênese. Este trabalho apresenta os resultados do estudo cariotípico de 51 casos de diferentes tipos de leu cemias. Os cromossomos foram obtidos através de cultura de células de sangue periférico, realizadas por 24 ou 48 horas, sem estimulação mitogênica. Em $74 \%$ dos pacientes foram observadas anomalias cromossômicas clonais como translocações, deleções, monossomias e trissomias. Muitas alterações foram compatíveis com outras previamente descritas e outras não, como a tran slocação en volven do os cromossomos 9 e 22, que origina o cromossomo Philadelphia e uma translocação complexa en volven do os cromossomos 4, 7 e11. Os resultados reforçam a importância da análise cromossômica em leucemia e seus ben efícios para o paciente.

Rev.bras.hematol.hemoter., 2000, 22(3): 374-386
\end{abstract}

Palavras-chave: Leucemia, citogenética, aberrações cromossômicas

\section{Introdução}

O câncer é uma doença genética (1) e as mutações que Ihe dão origem podem ser hereditariamente transmitidas pela linhagem germinativa ou adquiridas nos tecidos somáticos (2). Tais alterações no DNA podem ser causadas por fatores internos como, por exemplo, defeitos nos mecanismos de reparo ou replicação, ou externos, como radiação ionizante, mutagênicos químicos e biológicos (3).
Muitas evidências sugerem que a maioria dos neoplasmas são de natureza clonal, ou seja, surgem da expansão de uma única alteração celular inicial. Divisões celulares sucessivas podem produzir subpopulações de células filhas com alterações genéticas adicionais, em função de uma instabilidade genômica, que podem fornecer vantagens seletivas e culminar na aquisição da malignidade. Algumas destas alterações podem dar às células uma vantagem de

1 - Geneticista, Depto. de Biologia Molecular, FAMERP

2 - Mestranda, Depto. de Biologia, IBILCE-UNESP

3 - Hematologista, Depto. de Medicina, FAMERP

4 - Pediatra, Depto. de Pediatria, FAMERP

Correspondência para: Agnes Cristina Fett-Conte

Faculdade de Medicina de São José do Rio Preto

Av. Brigadeiro Faria Lima, 5416. Bairro Universitário. São José do Rio Preto. SP. CEP 15090-000

Fone: (17) 210-5000 r. 348

E-mail: genetica@famerp.br 
crescimento, por exemplo, criando tumores que contenham variantes clonais (4).

A carcionogênese, portanto, é vista como um processo genético de múltiplos passos, onde as células tumorais, gradual e progressivamente, adquirem um novo fenótipo característico, com um comportamento mais agressivo (5).

Os avanços da genética molecular e da citogenética têm aumentado a capacidade de detecção das alterações das células neoplásticas hematopoéticas e de tumores sólidos, possibilitando, em muitos casos, o esclarecimento dos mecanismos responsáveis pela etiologia e patogênese das neoplasias (3). Têm, inclusive, permitido o mapeamento de vários genes envolvidos nos processos neoplásicos, como oncogenes e genes supressores de tumor (6).

Neste contexto, as leucemias se destacam como um exemplo de doenças que apresentam alterações cromossômicas numéricas e/ou estruturais altamente consistentes e específicas, envolvendo genes que, uma vez alterados qualitativa ou quantitativamente, atuam como fatores de iniciação e progressão neoplásicas $(7,8)$.

A citogenética aplicada à clínica, inclusive, se iniciou nas leucemias, com a descoberta em 1960 do cromossomo Philadelphia (Ph), um marcador da leucemia mielóide crônica (9). Hoje, tem permitido a detecção de alterações cromossômicas como fator diagnóstico, a classificação citogenética das neoplasias hematológicas, a caracterização de diferentes estágios do desenvolvimento neoplásico, a avaliação da remissão, agudização e do prognóstico destas enfermidades e de diversos genes envolvidos nestes processos $(10,11,12)$.

No Hospital de Base de São José do Rio Preto, até o início do ano de 1999, não havia condições laboratoriais para realização de estudos moleculares e preparação de células da medula óssea para o estudo de leucemias. 0 estudo cromossômico do sangue periférico foi o primeiro passo para a posterior implantação das mesmas.

0 presente trabalho tem como objetivo apresentar as alterações cromossômicas observadas em células leucêmicas do sangue periférico de pacientes atendidos no referido hospital, portadores de diferentes tipos de leucemias, no período de 1984 a 1998.

\section{Casuística e métodos}

No período de outubro de 1984 a novembro de 1998 foram encaminhadas para o Laboratório de Genética da Faculdade de Medicina de Rio Preto (FAMERP), para estudo das alterações cromossômicas, 146 amostras de sangue periférico de pacientes portadores de diferentes tipos de leucemias. Até fevereiro de 1999 não haviam sido implantadas técnicas para estudo de células da medula óssea e de citogenética molecular, hoje disponíveis com um serviço específico para diagnóstico citogenético e molecular.

Das amostras recebidas, em 51 delas (35\%), houve sucesso quanto a obtenção de células em condições de análise e os resultados de todos os pacientes são apresentados. Destes, apenas - caso 1 foi analisado após tratamento quimioterápico, cujo término ocorreu dois anos antes do estudo. Todos os demais pacientes não haviam recebido tratamento quimio ou radioterápico prévio.

Foram, então, realizadas análises citogenéticas em células do sangue periférico de 51 indivíduos, 32 do sexo masculino e 19 do sexo feminino, portadores de diferentes tipos de leucemias, diagnosticados pelo Serviço de Hematologia do Hospital de Base da FAMERP. O diagnóstico das leucemias seguiu as especificações internacionais, que incluíram exame clínico completo, hemograma, mielograma, análise citoquímica e/ ou biópsia de medula óssea.

As células foram cultivadas segundo a técnica descrita por Moorhead e col. (13), com modificações. Para cada paciente, foram montados três ou quatro frascos de cultura contendo de 0,2 a $0,5 \mathrm{ml}$ de plasma, $5,0 \mathrm{ml}$ de

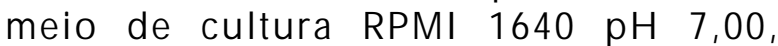
suplementado com $20 \%$ de soro fetal bovino ou soro humano do tipo $A B$ e solução de penicilina/estreptomicina $(100 \mathrm{U} / \mathrm{ml})$, sem a adição de fitohemaglutinina (estimulador mitogênico). 0 tempo de cultivo foi de 24 e/ou 48 horas e o número de células analisadas variou de cinco a sessenta e oito (Tabela 1 ).

Embora o número de metáfases obtidas tenha sido superior ao das estudadas, só foram selecionadas aquelas em condições de serem analisadas citogeneticamente. Os resultados 
foram comunicados para a equipe de hematologistas responsável pelos pacientes e sua aplicação clínica ou não seguiu os critérios adotados pela própria equipe.

As metáfases foram analisadas em bandamento GTG, segundo a técnica de Grouchy \& Turleau (14), com modificações e em coloração usual com Giemsa a $2 \%$. A classificação dos achados e os critérios para a determinação de clone seguiram as recomendações do ISCN (15).

\section{Resultados}

Foram analisados citogeneticamente 51 casos, dos quais 38 eram de leucemia mielóide crônica (LMC), nove de leucemia mielóide aguda (LMA) e quatro de leucemia linfocítica aguda (LLA).

O número cromossômico das amostras variou de 43 a 92 e a maioria das células apresentou número cromossômico normal. Foram encontradas alterações cariotípicas não clonais, ou seja, esporádicas, que compreenderam

Tabela 1. Idade dos pacientes, diagnóstico da leucemia, número de células analisadas e cariótipos obtidos

\begin{tabular}{|c|c|c|c|c|}
\hline Caso & $\begin{array}{l}\text { Idade } \\
\text { (anos) }\end{array}$ & Diagnóstico & $\begin{array}{l}\text { № células } \\
\text { Analisadas }\end{array}$ & Cariótipo \\
\hline 1 & 16 & LLA-L2 & 23 & 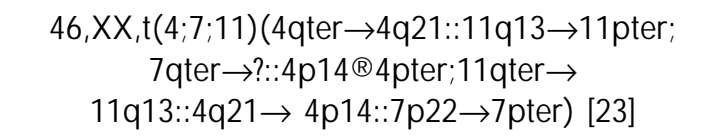 \\
\hline 2 & 32 & LMA-M2 & 20 & $46, X Y, t(8 ; 21)(q 22 ; q 22)[20]$ \\
\hline 3 & 71 & LMC & 12 & $46, X X, t(9 ; 22)(q 34 ; q 11), t(11 ; 19)(q 23 ; p 13)[12]$ \\
\hline 4 & 18 & LMA-M2 & 10 & $46, X X, t(8 ; 21)(q 22 ; q 22)[10]$ \\
\hline 5 & 34 & LMC & 10 & 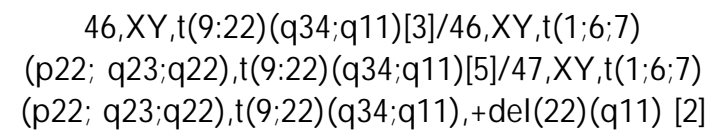 \\
\hline 6 & $\mathrm{NI}$ & LMA & 16 & $45 \sim 48, X Y,+4$, del(5)(p14@pter);+9;+mar;-17[cp 16] \\
\hline 7 & 51 & LMC & 07 & $46 \sim 50, X X, t(9: 22)(q 34 ; q 11),-22,+\operatorname{del}(22)(q 11)[c p 7]$ \\
\hline 8 & 02 & LLA & 06 & $44 \sim 46, X Y,+21 c[6]$ \\
\hline 9 & 07 & LMA & 19 & $45, X X,-8,-15,+\operatorname{mar}[19]$ \\
\hline 10 & 47 & LMC & 68 & $33 \sim 47, X X, t(9: 22)(q 34 ; q 11),+2 ;-5 ;-7 ;-8[c p 68]$ \\
\hline 11 & 34 & LMC & 15 & $\begin{array}{c}46, X Y, t(9 ; 22)(q 34 ; q 11)[13 \mathrm{~V} \\
47, X Y, t(9 ; 22)(q 34 ; q 11),+\operatorname{del}(22)(q 11)[2]\end{array}$ \\
\hline 12 & 76 & LMC & 11 & $45, X,-Y, t(9 ; 22)(q 34 ; q 11)[11]$ \\
\hline 13 & 02 & LLA & 15 & $47, X Y,+21 c[15]$ \\
\hline 14 & 39 & LMC & 10 & $\begin{array}{c}46, X X, t(9 ; 22)(q 34 ; q 11)[8] \\
47, X X, t(9 ; 22)(q 34 ; q 11),+\operatorname{del}(22)(q 11)[2]\end{array}$ \\
\hline 15 & 45 & LMC & 32 & $\begin{array}{l}46, X Y, t(9 ; 22)(q 34 ; q 11)[29] \\
45, X,-Y, t(9 ; 22)(q 34 ; q 11)[3]\end{array}$ \\
\hline 16 & 24 & LMC & 10 & $46, X X, t(9 ; 22)(q 34 ; q 11)[10]$ \\
\hline 17 & 50 & LMC & 10 & $46, X X, t(9 ; 22)(q 34 ; q 11)[10]$ \\
\hline 18 & 32 & LMC & 12 & $46, X X, t(9 ; 22)(q 34 ; q 11)[12]$ \\
\hline 19 & 06 & LMC & 08 & $46, X X, t(9 ; 22)(q 34 ; q 11)[08]$ \\
\hline 20 & $\mathrm{NI}$ & LMC & 10 & $46, X X, t(9 ; 22)(q 34 ; q 11)[10]$ \\
\hline
\end{tabular}




\begin{tabular}{|c|c|c|c|c|}
\hline Caso & $\begin{array}{l}\text { Idade } \\
\text { (anos) }\end{array}$ & Diagnóstico & $\begin{array}{l}\text { № células } \\
\text { Analisadas }\end{array}$ & Cariótipo \\
\hline 21 & 20 & LMC & 14 & $46, X X, t(9 ; 22)(q 34 ; q 11)[13]$ \\
\hline 22 & 66 & LMC & 11 & $46, X X, t(9 ; 22)(q 34 ; q 11)$ [11] \\
\hline 23 & 54 & LMC & 17 & $46, X X, t(9 ; 22)(q 34 ; q 11)[17]$ \\
\hline 24 & 48 & LMC & 15 & $46, X Y, t(9 ; 22)(q 34 ; q 11)[15]$ \\
\hline 25 & 46 & LMC & 15 & $46, X Y, t(9 ; 22)(q 34 ; q 11)[15]$ \\
\hline 26 & 13 & LMC & 05 & $46, X Y, t(9 ; 22)(q 34 ; q 11)[05]$ \\
\hline 27 & 61 & LMC & 15 & $46, X Y, t(9 ; 22)(q 34 ; q 11)[15]$ \\
\hline 28 & 34 & LMC & 13 & $46, X Y, t(9 ; 22)(q 34 ; q 11)[13]$ \\
\hline 29 & 41 & LMC & 12 & $46, X Y, t(9 ; 22)(q 34 ; q 11)[12]$ \\
\hline 30 & 79 & LMC & 07 & $46, X Y, t(9 ; 22)(q 34 ; q 11)[07]$ \\
\hline 31 & 36 & LMC & 10 & $46, X Y, t(9 ; 22)(q 34 ; q 11)[10]$ \\
\hline 32 & 52 & LMC & 10 & $46, X Y, t(9 ; 22)(q 34 ; q 11)[09]$ \\
\hline 33 & 67 & LMC & 11 & $46, X Y, t(9 ; 22)(q 34 ; q 11)[11]$ \\
\hline 34 & 16 & LMC & 15 & $46, X Y, t(9 ; 22)(q 34 ; q 11)[15]$ \\
\hline 35 & 23 & LMC & 10 & $46, X Y, t(9 ; 22)(q 34 ; q 11)[10]$ \\
\hline 36 & 91 & LMC & 06 & $46, X Y, t(9 ; 22)(q 34 ; q 11)[06]$ \\
\hline 37 & 54 & LMC & 15 & $46, X Y, t(9 ; 22)(q 34 ; q 11)[15]$ \\
\hline 38 & 66 & LMC & 05 & $46, X Y, t(9 ; 22)(q 34 ; q 11)[05]$ \\
\hline 39 & 52 & LMC & 06 & $46, X Y, t(9 ; 22)(q 34 ; q 11)[06]$ \\
\hline 40 & 39 & LMC & 17 & $46, X Y, t(9 ; 22)(q 34 ; q 11)[17]$ \\
\hline 41 & 53 & LMC & 10 & $46, X Y$ \\
\hline 42 & $01 \mathrm{~m}$ & LLA & 16 & $46, X X$ \\
\hline 43 & 60 & LMA & 08 & $46, X Y$ \\
\hline 44 & 76 & LMC & 11 & $46, x x$ \\
\hline 45 & 63 & LMA & 10 & $46, X X$ \\
\hline 46 & 80 & LMC & 05 & $46, X Y$ \\
\hline 47 & 29 & LMA & 09 & $46, X Y$ \\
\hline 48 & 53 & LMA & 09 & $46, X Y$ \\
\hline 49 & $\mathrm{NI}$ & LMA & 06 & $46, X Y$ \\
\hline 50 & 53 & LMC & 15 & $46, X Y$ \\
\hline 51 & 76 & LMC & 07 & $46, X Y$ \\
\hline \multicolumn{5}{|c|}{$\begin{array}{l}\text { LMA = leucemia mielóide aguda } \\
\text { LMC = leucemia mielóide crônica } \\
\text { LLA = leucemia linfocítica aguda } \\
\text { LLC = leucemia linfocítica crônica } \\
\mathrm{NI}=\text { não informada } \\
\mathrm{M}=\text { idade em meses }\end{array}$} \\
\hline
\end{tabular}




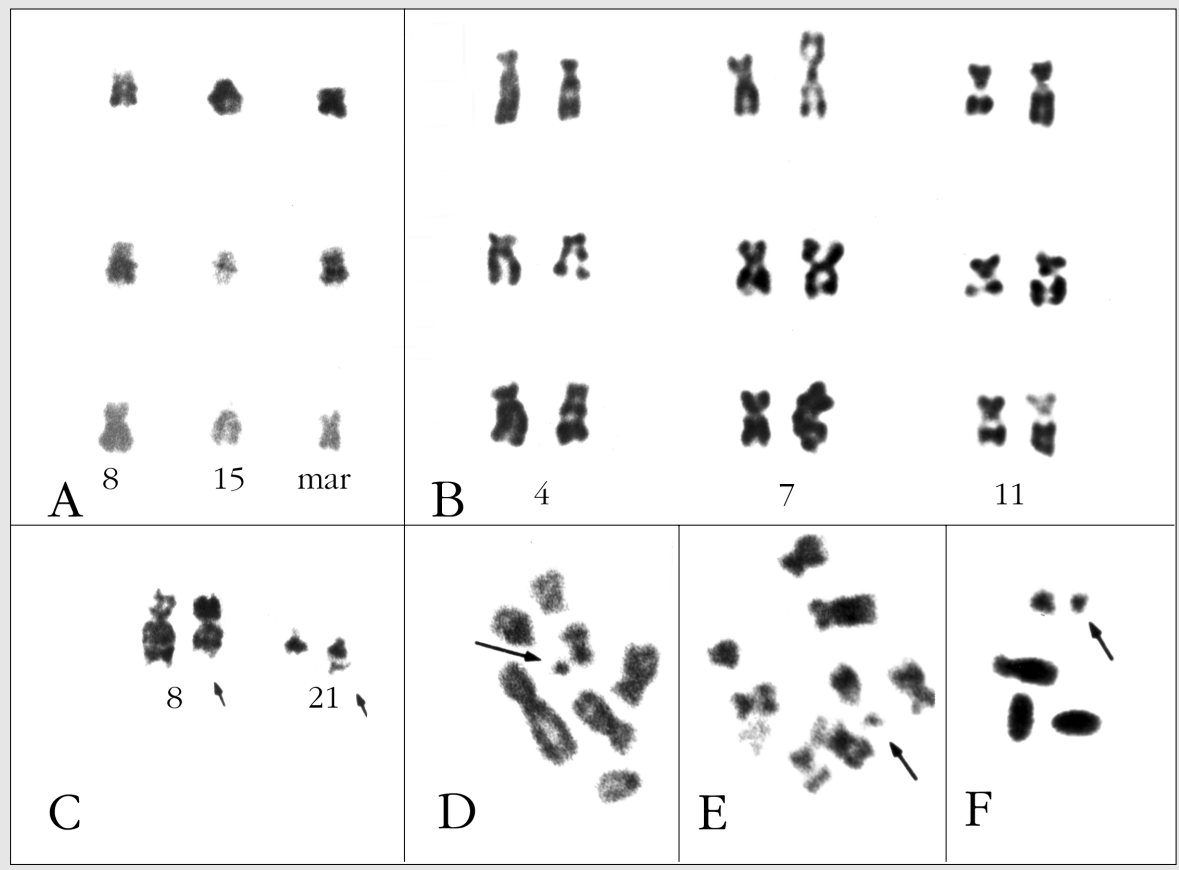

Figura 1. Cariótipos parciais mostrando a monossomia dos cromossomos 8 e 15 e o cromossomo marcador (A), a translocação envolven do os cromossomos 4, 7 e 11, com cromossomos normais à esquerda (B), a translocação entre os cromossomos 8 e 21 (C) e metáfases parciais com as setas indicando o cromossomo $\mathrm{Ph}(\mathrm{D}-\mathrm{F})$.

translocações, trissomias, monossomias totais ou parciais e cromossomos marcadores. Onze casos (41-51) apresentaram células com cariótipos normais, ao nível de resolução das técnicas utilizadas. Destes, cinco eram LMC, cinco LMA e um LLA. Em 40 casos (78,4\%) foram encontradas alterações clonais.

A idade dos pacientes, o diagnóstico, o número de metáfases analisadas e os cariótipos com aberrações cromossômicas clonais, de cada caso, estão apresentados na Tabela 1. Nesta tabela, para facilitar a visualização dos achados, os códigos de 1 a 15 correspondem aos casos com alterações diversas e mais complexas, seguidos daqueles com apenas a $t(9 ; 22)$ (16 a 40) e dos normais (41 a 51). A Figura 1 mostra cariótipos e metáfases parciais de algumas destas alterações.

Os casos 8 e 13 eram pacientes portadores de síndrome de Down, nos quais a trissomia simples do cromossomo 21 foi confirmada na linhagem constitucional, que foi obtida de cultura de linfócitos do sangue periférico com estimulação mitogênica. As células destes pacientes quando cultivadas em meio sem estimulação mitogênica, apresentaram a própria trissomia como a única alteração encontrada. No caso 8, embora todas as metáfases apresentassem a trissomia do 21, elas também mostravam monossomias não clonais envolvendo praticamente todos os cromossomos.

\section{Discussão}

As alterações cromossômicas numéricas e/ ou estruturais consistentes e específicas em leucemias, além de contribuírem para a compreensão da base genética destas patologias, têm importância fundamental no diagnóstico e prognóstico dos pacientes (16). 0 diagnóstico preciso e a classificação celular exata, são essenciais para o sucesso do tratamento e para o estudo dos processos biológicos destas doenças $(2,17)$.

Por exemplo, o paciente com LMC e cromossomo Ph no cariótipo, apresenta evolução clínica melhor, sobrevida maior e prognóstico 
mais favorável do que aquele sem o Ph que, geralmente, responde mal ao tratamento e apresenta uma sobrevida mais curta $(18,19,20)$.

$\mathrm{O}$ cromossomo $\mathrm{Ph}$, que deriva da $\mathrm{t}(9 ; 22)$, é observado em cerca de $90-95 \%$ dos casos de LMC (21). Neste estudo, este cromossomo derivado apareceu em $86,84 \%$ dos casos de LMC. Nos casos 5, 7, 11 e 14 foi observado, inclusive, em duplicata. Portanto, a proporção de casos de LMC com e sem o Ph, aqui obtida, foi semelhante às já descritas anteriormente $(22,11,23,24)$.

Como consequência da translocação envolvendo os cromossomos 9 e 22, o oncogene $\mathrm{c}-\mathrm{ABL}$, mapeado em 9q34, se funde com uma pequena região de seis quilobases $(k b)$ do cromossomo 22, designada como região de ponto de quebra ou gene BCR. Essa translocação resulta em uma nova proteína quimérica com atividade tirosina quinase e potencial transformante $(18,25,26,27,28,29)$.

Embora seja muito mais frequente nos casos de LMC, a t $(9 ; 22)$ é observada em muitos tipos de leucemias e de outras neoplasias (25). Sua participação efetiva na patogênese dos casos aqui estudados é certa e sua presença confirma o diagnóstico hematológico e sugere um prognóstico mais favorável para os seus portadores.

Cinco pacientes $(13,16 \%)$ com LMC apresentaram cariótipo normal. Deve ser considerado o fato de terem sido estudadas células do sangue periférico e não da medula óssea, o que sugere que a frequência de LMC e das outras leucemias, com cariótipo normal, possa estar superestimada. Além disso, as células analisadas poderiam ser linfoblastos, portanto não representativos da linhagem leucêmica. Uma vez que as células da medula geralmente revelam mais alterações cariotípicas, tais células devem ser prioritariamente estudadas. Por outro lado, deve ser também salientado que LMC com cariótipo normal é esperado em 5 a 10\% dos casos e reflete um prognóstico ruim (9). Contudo, nem sempre isso é verdadeiro. 0 cariótipo normal não exclui a presença de aberrações cromossômicas submicroscópicas, que não podem ser observadas com as técnicas da citogenética clássica, como as utilizadas neste estudo. Por exemplo, Estop e cols. (30) descreveram um paciente com LMC e cariótipo normal, cuja análise citogenética molecular, pela técnica de FISH, detectou o rearranjo $B C R / A B L$. Esse caso Ph-negativo e BCR/ABL positivo resultou da inserção da sequência do cromossomo 9 no cromossomo 22, sem alterar a morfologia cromossômica.

Nos casos de LMC em que outras alterações clonais atípicas ou adicionais foram observadas, como nos casos 3, 5, 7, 10,11, 12, 14 e 15 pode ser sugerida uma progressão clonal das células leucêmicas. A crise blástica, que advém de tal progressão, é uma fase altamente maligna e revela um estágio mais avançado da doença. Sua detecção ou prevenção reflete diretamente na terapia que deve ser utilizada (25).

Em LLA, a hiperdiploidia, com mais de 50 cromossomos, é a anomalia citogenética mais comumente observada e está relacionada a um prognóstico bom (31). Paszek-Vigier e cols. (32) relataram 72 pacientes em que o ganho de cromossomos foi muito mais frequente do que a perda e foi associado com prognóstico excelente. Por outro lado, a monossomia do cromossomo 7 e a inversão paracêntrica do cromossomo 3, envolvendo as bandas $3 q 21$ e $3 q 26$, têm sido descritas em muitos casos e estão relacionadas a um prognóstico ruim, pois acarretam maior resistência a quimioterapia $(33,34)$.

É interessante que em LLA é observada de forma consistente a $t(4 ; 11)(q 21 ; q 23), 0$ envolvimento da banda 11q23 e do cromossomo 7 nas alterações primárias e secundárias já descritas $(35,36,37)$. A t $(4 ; 11)$ é detectada tanto nas células com morfologia típica de L1 quanto de L2 e também já foi observada excepcionalmente em células L3 (38). Quanto aos aspectos imunofenotípicos, podem ser caracterizadas como pré-B, bifenotípicas e de linhagem mista (35). As células com esta translocação não só apresentam dificuldade na identificação de sua natureza, como a frequência das mesmas varia nos diferentes estudos relatados (25).

Na região 11q23 está mapeado o gene $M L L$ (ALL-1 ou HRX), um proto-oncogene cujo envolvimento é o mais importante, não só na $\mathrm{t}(4 ; 11)$ como em outras translocações, associadas a diferentes leucemias e outras neoplasias, que envolvem esta região e uma variedade de outros cromossomos $(25,39)$. O proto-oncogene MLL codifica uma proteína que parece ter um papel na regulação da transcrição (35). No 
cromossomo 4, por sua vez, na região envolvida na translocação, está mapeado o gene AF4 (FEL) que parece codificar um fator transcricional. Um possível gene quimérico resultante da fusão do MLL e do AF4, no derivado do cromossomo 11 seria o responsável pela patogênese da neoplasia (39). Em 11q23 também foi mapeado o oncogene ETS-1 que parece ser ativado quando translocado para $4 q 21$ (40). Os fatores de transcrição ETS representam uma família de genes envolvidos no controle da proliferação celular e no processo tumoral (41).

Assim, a presença da $t(4 ; 11)$ em LLA está associada a um prognóstico ruim (42).

0 envolvimento do cromossomo 7 tem sido relatado em anormalidades numéricas e estruturais observadas em diferentes neoplasias, inclusive, hematopoéticas. Deleções ou monossomias são consistentes em síndromes mielodisplásicas e em LMA e, nas primeiras, associadas à exposição anterior a agentes carcinogênicos ou leucemogênicos e a um prognóstico desfavorável $(33,43)$. Translocações envolvendo ambos os braços do 7 também são comuns em LMA e LLA e, neste cromossomo, já foram mapeados os genes TCR- $\beta$ e HOXA 9 (28).

0 caso 10 (LMC) apresentou monossomia do cromossomo 7. Há sugestão de que esta monossomia pode ter uma função específica no desenvolvimento e evolução de leucemias, o que já foi observado em LMA (44).

0 caso 3 (LMC) apresentou a $\mathrm{t}(11 ; 19)(q 23 ; p 13)$ adicional ao cromossomo $\mathrm{Ph}$. Essa translocação tem sido especialmente observada em LLA e promove a fusão gênica MLL-ENL, cujo transcrito varia com a idade e imunofenótipo e atua como fator transcricional (45).

0 caso 1 apresentou um rearranjo mais complexo, envolvendo os cromossomos 4, 7 e 11. Nestes cromossomos, nas bandas onde ocorreram as quebras, ou próximas a elas, já foram também mapeados os oncogenes BCL-1, KIT, ARA-F2, ERGF, MEN1, PLZF, RAL-A e MET, o supressor de tumor ST3 e o gene da Neoplasia Endócrina Múltipla do tipo 1 (46, 40, 47, 48).

A ausência de estudos moleculares impede a confirmação do envolvimento destes genes no processo neoplásico dos pacientes aqui estudados, embora este não possa ser descartado.
Inclusive, a alteração encontrada no caso 1 pode não refletir o clone primário, pois este paciente foi o único da amostra que recebeu tratamento quimioterápico anterior à época do estudo, 0 que pode predispor a alterações secundárias (49). Contudo, o fato de não terem sido observadas outras alterações clonais não descarta a possibilidade de que se trate do clone primário.

Os pacientes 8 e 13 tinham síndrome de Down (SD) e a trissomia do cromossomo 21 foi a única alteração encontrada nas células estudadas. A SD está associada a um risco aumentado de desenvolver câncer (seis vezes maior que a população geral), especialmente neoplasias hematopoéticas (10-20 vezes maior), contudo a base desta predisposição não é conhecida $(50,51,52)$. Além disso, nos primeiros meses de vida, os pacientes tendem a desenvolver uma alteração mieloproliferativa transitória, reconhecida como leucemia transitória ou mielopoese anormal transitória (MAT), que regride espontaneamente durante os primeiros meses de vida. Cerca de $25 \%$ destes casos desenvolvem leucemias agudas até os três anos de idade (53). A maioria das células na MAT não apresenta alterações cariotípicas adicionais à trissomia do 21, diferentemente daquelas das leucemias. Portanto, a proliferação anormal das células pode ser relacionada à cópia extra do 21. As alterações cariotípicas adicionais podem ser as responsáveis pela recaída da doença, sugerindo, assim, que a trissomia é o fator predisponente e que outros eventos podem ser requeridos antes do processo leucêmico (50). É interessante que em indivíduos sem SD, portadores de LLA-L1 e L2 a trissomia do 21 pode ser observada como única anomalia ou como anomalia adicional em cerca de $3 \%$ dos casos e, naqueles com SD portadores de leucemias, pode ser a única alteração encontrada (25).

É referida, inclusive, a participação de alguns genes mapeados em 21q, como o AML1 na patogênese das leucemias meilóides e linfóides, cujos produtos parecem atuar na hematopoese normal $(50,45)$. Mutações submicroscópicas envolvendo estes genes poderiam explicar os casos de leucemia em SD sem alterações adicionais à trissomia do 21. Além disso, nos casos de SD aqui descritos, as células estudadas poderiam não ser representativas do 
clone leucêmico. Esta mesma possibilidade existe para aqueles casos (41-51) que apresentam todas as células normais, como já referido anteriormente.

Os casos 2 e 4 mostraram a anomalia mais freqüentemente envolvida na LMA-M2, a t(8;21), que está associada a um prognóstico favorável. 0 gene quimérico resultante AML1/ETO produz uma proteína de fusão AML/ETO, que parece ser capaz de induzir a transformação leucêmica alterando o ciclo celular e o processo de apoptose, através da interação com genes normalmente regulados pelo AML1 $(54,55,27$, $12,45)$. No caso 9, o cromossomo marcador sugere ser um cromossomo $8 \mathrm{com}$ deficiência intersticial do braço longo. Segundo Govaerts e cols. (56) a deficiência parcial ou total deste cromossomo é fundamental para 0 desenvolvimento da LMA. Além disso, a monossomia do 15 , como observado no mesmo caso, já foi descrita em vários casos de LMA e parece conferir um comportamento mais agressivo para a doença (57).

A nulissomia do cromossomo $Y$ tem sido a alteração numérica mais comum nas lesões benignas e malignas de próstata, em outras lesões malignas e também muito freqüente em LMA $(58,59,11)$. Segundo Herens et al. (60), o cariótipo 45,x é observado em células de medula óssea de homens idosos, mas também em malignidades hematológicas. Se essa perda em células neoplásicas está relacionada ao processo de envelhecimento ou é parte do processo carcinogênico, ainda não se sabe. Estes autores encontraram a nulissomia do $Y$ em sete casos de leucemias, três dos quais foram considerados como evento secundário em pacientes com LMA-M2. Em três outros casos os autores referiram que esta alteração era parte de um cariótipo complexo e somente em um paciente foi observado o cariótipo 45, $x$ sem qualquer outro rearranjo, o que pôde ser positivamente correlacionado com o processo neoplásico. Krauter et al. (44) encontraram perda de $Y$ como única anomalia em $1 \%$ dos casos de leucemias analisados.

$\mathrm{Na}$ amostra aqui relatada, os casos 12 e 15, com 76 e 45 anos, respectivamente, apresentaram a perda do $Y$ como uma alteração adicional à presença da $t(9 ; 22)$. No caso 12 uma relação com a idade do paciente pode ser sugerida, embora o fato de todas as 11 células estudadas apresentarem a mesma alteração possa sugerir a participação da mesma no processo leucêmico.

A $t(1 ; 6 ; 7)$, observada no caso 5 , pode ser relacionada à evolução clonal da doença por ter sido adicional ao Ph. Specchia et al. (61) relatam que translocações envolvendo os cromossomos 1 e 7 são relativamente raras em neoplasias mielóides, sendo encontradas em menos de $1 \%$ dos casos. O rearranjo que ocorre mais freqüentemente consiste de uma translocação não balanceada $\mathrm{t}(1 ; 7)(\mathrm{p} 11 ; \mathrm{p} 11)$, que resulta em uma perda completa de $7 q$, associada com uma resposta à terapia, ou a exposição a agentes ambientais causadores de mieloproliferação. Esses autores observaram em três casos de LMA uma translocação balanceada $t(1 ; 7)$ (p36;q34), não relatada previamente. 0 caso aqui estudado apresentou o envolvimento do cromossomo 6 além do 1 e do 7 e muitas translocações envolvendo o cromossomo 6 têm sido descritas em condições hematológicas malignas $(62,63$, $64,65,66)$. Inclusive, algumas deleções envolvendo, a região $6 q 25$-27 estão associadas com linfomas de diferentes graus (67).

Todas as demais alterações numéricas e estruturais encontradas nos outros casos analisados envolveram cromossomos e/ou regiões cromossômicas cujo envolvimento é freqüentemente descrito nas mutações gênicas e cromossômicas observadas em leucemias e em outras condições malignas, podendo ser destacadas a trissomia do cromossomo 2 e a monossomia do $17(57,59,25,68)$.

Nas alterações previamente descritas, aqui citadas, os autores relatam o envolvimento de oncogenes e supressores de tumor que podem ter participado também da patogênese dos casos aqui estudados. Por outro lado, embora o estudo das células neoplásicas através das técnicas citogenéticas convencionais seja muito útil, como mostram os resultados apresentados, outras alterações podem ser detectadas e outras informações obtidas, com o estudo molecular destas células, especialmente da medula óssea, 0 que deve ser priorizado, inclusive, com a utilização da técnica de "Fluorescence in situ Hybridization" (FISH) $(23,69,70,71,72)$ e PCR $(73,37)$. 


\section{Conclusões}

A leucemia é uma doença genética na qual várias mutações participam do processo maligno, muitas das quais possíveis de serem diagnosticadas em estudos citogenéticos. Cada alteração pode estar associada aos eventos de iniciação ou de progressão neoplásica.

Este trabalho mostrou a importância do estudo cromossômico em leucemias, inclusive muitas das alterações encontradas foram úteis para entendimento do processo maligno das células estudadas. Além disso, evidenciou a necessidade da implantação de outras técnicas complementares para 0 diagnóstico genético, incluindo o estudo em medula óssea e técnicas moleculares.

\section{Chromosomal study of peripheral blood in} patients with different types of leukemiafrom Hospital de Base, São José do Rio Preto - SP

Agnes C. Fett-Conte, Cristina B. VendrameGoloni, Carlos M. Homsi, Leila N.B. Borim, Paulo A. Zola, Octávio Ricci

\section{Summary}

The analysis of chromosomal changes in leukemia has had a direct involvement in the diagnosis, prognosis and treatment of patients. Furthermore, it has allowed the understanding of biological processes involved in carcinogenesis. This work presents the results of a kariotypic study of 51 cases of different types of leukemia. The chromosomes were obtained through a 24 or 48 hour culture of cells of the peripheral blood with no mitogenic stimulation. In $74,5 \%$ of patients clonal chromosomic abnormalities wereobserved such as translocations, deletions, monosomies and trisomies. Many of the alterations were similar to results previously published and others were not such as the translocation in volving the chromosomes 9 and 22, which leads to the Philadelphia chromosome and the complex translocation involving the chromosomes 4, 7 and 11 . The results emphasized the importance of chromosomal analysis in leukemia, its ben efits to the patient and to the knowledge of the biological mechanisms involved in this pathology.

Rev.bras.hematol.hemoter.,2000, 22 (3): 374-386
Key words: Leukemia, cytogenetic, chromosomic aberrations

\section{Referências Bibliográficas}

1. Robbins S.L., Cotran R.S., Kumar V. Fundamentos de Robbins: patologia estrutural efuncional. $\mathbf{5 e d}$. Rio deJaneiro: Guanabara Koogan, 1996, p. 605.

2. Isselbacher K.J., Braunwald E., Wilson J.D., Martin J.B., Fauci A.S., Kasper D.L. Medicina Interna, 13 ed. México: Nueva Editorial Interamericana, 1995, v. 1, p. 391-409.

3. Vogel F., Motulsky A.G. Human Genetics: Problems and approaches, Ed Springer, 1997, p.851.

4. Hall I., Gioli D.D., Weissman B.E., Testy T.D. Identification of additional complemantation groups that regulate genomic instability. Genes, Chromosome \& Cancer, 1997; 20: (2): 102-112.

5. Pejovic T., Heim S., Orndal J.Y., Mandahl N.,

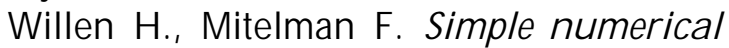
chromosomeaberrationsin well- differentiated malignant epithelial tumors. Cancer, Genet. Cytogenet, 1990; 49: 95-101.

6. Fejzo M.S., Godfrey T., Chen C., Waldman F., Gray J.W. Molecular Cytogenetic of consistent abnormalities at 8q 12-q22 in breast cancer. Genes, Chromosomes \& Cancer, 1998; 22: 105-113.

7. Popescu N.C., Zimonjic D.B. Molecular cytogenetic characterization of cancer cell alterations. Cancer Genet. Cytogenet. 1997; 93: 10-21.

8. Mühlman J., Thaler J., Hilbe W., Bechter 0 ., Erdel M., Utermann G., Duba H.C. Fluorescence in situ Hybridization (FISH) on peripheral blood smears for monitoring Philadelphia chromosome-positive chronic myeloide leukemia (LMC) during interferon treatment - A new strategy for remission assessment. Genes, Chromosome \& Cancer, 1998; 21: 90-100.

9. Seebach C., Sanz P., Soto V., Fuenzalida P., Tapia S., Loredo E. Estudio citogenetico de leucemias. Bol.Hosp.Vinã del Mar, 1992; 48 (3-4): 188-194.

10. Mrózek K., Heinonen K., Chapelle A. de LA., Bloomfield C., Clinical sisgnificance of 
cytogen etics in acute myeloid leukemia. Sem. Oncol., 1997; 24 (1): 17-31.

11. Perkins D., Shawn B., Carstairs K., Bailey D., Pantalony D., Poon A., Fernandes B., Dubé I. Regional cancer cytogen etics: A report on 1.143 diagnostic cases. Cancer, Genet Cytogenet 1997; 96: 64-80.

12. Paskulin G.A., Philips G., Morgan R., Sanderberg A., Richkind K., Borovik C., MacGavran L. Rabinovich N., Erickson P., Drabkin $\mathrm{H}$. , Varela-Garcia M. Pre-clinical evaluation of probes to detect $t(8 ; 21)$ AML minimal residual disease by fluorescence in situ Hybridization. Genes, Chromosome \& Cancer, 1998; 21 (2): 70-85.

13. Moorhead P.S., Nowell P.C., Mellman W.J., Battips D.M., Hungerfor D.A. Chromosome preparations of leukocytes cultured from human peripheral blood. Exp. Cell Res., 1960; 20: 613-616.

14. Grouchy J.D.E., Turleau C., Atlas de maladies chromosomiques. Paris Expasion Scientifique Francise, 1997.

15. ISCN: An international system for human cytogenetic nomenclature. Basel: Karger, 1995, p. 114.

16. Johansson B., Brondum-Willsen, Billström R., Schiodt I., Mitelaman F. Translocation between the long arm of chromosomes 1 and 5 in hematologic malignancies are strongly associated with neoplasms of the myeloid lineages. Cancer, Genet. Cytognet, 1997; 99: 97-101.

17. Pui C.H. Recent advances in childhood acute Iymphoblastic leukemia. In: Temas de Hematologia, Belo Horizonte, 1997, p. 9-12.

18. Rowley J.D. Chromosome abn ormalites in leukemia. J. Clin. Oncol. 1988; 6: 194-202.

19. Wyngaarden J.B., Smith Jr. L.H. Cecil: Tratado de medicina interna $\mathbf{1 8}$ ed. Rio de Janeiro: Guanabara, 1990; 1: 585-886.

20. Aurich J., Dastugue N., Duchayne E., Schlaifer D., Rigal-Huguet F., Caballin M.R. Location of the BCR-ABL fusion gene on the 9q34 band in two cases of Ph-positive chronic myeloid. Genes, Chromosomes \& Cancer, 1997; 20: 148-154.

21. Rowley J.D. A new consistent chromosomal abnormality in chronic myelogenous leukaemia identifield by quinacrine fluorescence and giemsa staining. Nature 1973; 243: 290-293.

22. Eibl B., Ebner S., Duba C., Böck G., Romani N., Erdel M., Gächter A., Niederwieser D., Schuler G., Dendrictic cells generated from blood precursors of chronic T-cel response. Genes, Chromosomes \& Cancer, 1997; 20: 215-223.

23. Abruzzese E., Pettenati M.J., Cox K., Jackle B., Watts R.G., Carroll A.J., Steuterman M.C., Rao P.N. Identification of BCR-ABL fusion on chromosome 9 by Fluorescence in Situ Hybridization in two chronic myeloid leukemia cases. Cancer, Genet. Cytogenet, 1998; 105: 164-167.

24. Grand F.H., Chase A., Iqbal S., Nguyen D.X., Lewis J.L., Marley S.B., Davidson R.J., Goldman J.M., Gordon M.Y. A two-color $B C R-A B L$ probe that greatly reduces the false positive and false negative rates for Fluorescencein situ Hybridization in chronic myeloid leukemia. Genes, Chromosomes \& Cancer, 1999; 23: 109-115.

25. Heim S., Mitelman F., Chromosomal and molecular gen etic aberration of tumor cells. New York: Wiley-Liss, 2. Ed., 1995.

26. Aziz K.J. Clinical molecular biology: concepts and applications.Adv.Clin,Chem, 1996; 32: 39-72.

27. Peralta-Zaragoza O., Bahena-Roman M., Díaz-Benítez C.E. Madrid-Marina V. Regulacíon del ciclo celular y desarrollo de cáncer: perspectivas terapéuticas. Salud Pública Méx. 1997; 39: 451-460.

28. Sánches-Garcia. Consequences of chromosomal Abnormalities in tumor development. Annu. Rev. Genet, 31: 429-453.

29. Wlodarska I., Pittaluga S., Syul M., Martiat P., Dierlamm J., Michaux L., Wolf-Peeters C., Cassiman J.J., Mecucci C., Berghe H. Philadelphia-like translocation $\mathrm{t}(9 ; 22)$ (q34q11) found in a follicular lymphoma involving not bcr and abl but igl-mediated rearrangement of an unknow gene on 9q34. Genes, Chromosomes \& Cancer 1997; 20: 113-119.

30. Estop A.M., Sherer C., Cieply K., Groft D., Burcoclu A., Jhanwar S., Thomas J. A Phnegative chronic myeloid leukemia Patient with a now-classical BCR-ABL 
rearrangement characterizes by Fluorescence in situ Hybridization. Cancer Genet. Cytognet, 1997; 96: 174-176.

31. Nordgren A., Nordenskjöld M., Söderhäld S., Porwit-MacDonald A., Blennow E. A case of acutelymphoblastic leukemia, near-triploidy, and poor autcome: characterization by Fluorescence in situ Hybridization using chromosome-specific libraries from all human chromosomes. Cancer, Genet Cytogenet. 1997; 99 (2): 93-96.

32. Paszek-Vigier M., Talmant $P$., Mechinaud F., Garand R., Harousseau J.L., Bataille R., AveetLoiseau $H$. Comparative genomic hybridization is a powerful too, compelmentary to cytogenetics, to identify chromosomal abnormalities in childhood acute Iymphoblastic leukemia. Br. J. Haematol. 1997; 99: 589-596.

33. Pedersen B., Koch J., Hansen K.B., Hindkjar J., Andersen C.L. The monosomy 7 clone interphase and metaphase cell populations: A combined chromosome and primed in situ labeling. Acta Haematol. 1997; 97: 216-221.

34. Shi, G.,Weh, H.J., Dührsen, U., Zeller, W., Hossfeld, D.K. Chromosomal abnormality inv(3)(q21q26) associated with multilineage hematopoietic progenitor cells in hematopoietic malignancies. Cancer, Genetics and Cytogenetic, 1997; 96: 58-63.

35. Faderl S., Kantarjian H.M., Talpaz M., Estrov Z. Clinical Significance of cytogenetic abnormalities in adult aacutelymphoblastic leukaemia. Blood, 1998; 91: 3995-4019.

36. Forrest D.L., Nevill T.J., Horsman D.E., Brockington D.A., Fung H.C., Toze C.L., Conneally E.A., Hogge D.E., Sutherland, H.J., Nantel S.H., Shepherd J.D., Barnett M.J. Bone marrow transplantation for adults with acute leukaemia and 11q23 chromosomal abnormalities. British Journal of Hematology, 1998; 103: 630-638.

37. Reichel M., Gillert E., Breitenlohner I., Repp R., Greil J., Beck J.D., Marschalek R. Rapid isolation of chromosomal breakpoints from patients with $\mathrm{t}(4 ; 11)$ acute lymphoblastic leukaemia: implications for basic and clinical research. Cancer Res., 1999; 59 (14): 3357-3362.
38. Ferro M.T., San Roman C., Munoz A., Madero L., Olmeda F., Fernandez-Ranada J.M., Mecucci C. Translocation $(4 ; 11)$ in ALL: a new morphologic aspect? Cancer Genet. Cytogenet, 1986; 23 (2): 145-149.

39. Butler, L.H., Slany, R., Cui,X., Cleary, M.L., Mason, D.Y. The HRX proto-oncogene product is widely expressed in human tissues and localizes to nuclear structures. Blood, 1997; 89 (9): 3361-3370.

40. McApline P.J., Stranc L.C., Boucheix C., Shows T.B., The 1990 catalog of mapped genes and report of the nomenclature committee. Cytogenet. Cell Genet, 1990; 55: 5-76.

41. Zhou J., AY NG., Tymms M.J., Jermiin L.S., Seth A.K., Thomas R.S., Kola I. A novel transcription fator, ELF5, belongs to the ELF subfamily of ETS genes and maps to human chromosome11p13-15, a region subjectto $\mathrm{LOH}$ and rearrangement in human carcinoma cell lines. Oncogene, 1998; 17: 2719-2732.

42. Satake N., Maseki N., Nishiyama M., Kobayashi H., Sakurai M., Inaba H., Katano N., Horiskoshi Y., Eguchi H., Miyake M., Seto M., Kaneko Y. Chromosome abnormalities and MLL rearrangements in acute myeloid leukaemia of infants. Leukaemia, 1999; 13: 1013-1017.

43. Dabaja B.S., Faderl S., Thomas D., Cortes J., O'Brien S., Nasr F., Pierce S., Hayes K., Glassman A., Keating M., Kantarjian H.M. Deletions and losses in chromosomes 5 or 7 in adult acute lymphocytic leukaemia: incidence, associations and implications. Leukaemia, 1999; 13 (6): 869-872.

44. Krauter J., Ganser A., Bergmann L., Raghavachar A., Hoelzer D., Lubbert M., Schlimok G., Arnold R., Kirchner H., Port M., Heil G. Association between strutural and numerical chromosomal aberration in acute myeloblastic leukemia: a study by RT-PCR and FISH in 447 patients with de-novo AML. Ann. Hematol, 1999; 78: 265-269.

45. Rubnitz J.E., Camitta B.M., Mahmoud H., Raimondi S.C., Carrol A.J., Borowitz M.J., Shuster J.J., Link M.P., Pullen D.J., Downing J.R., Behm F.G., Pui C.H. Childhood acute lymphoblastic leukemia with the MLL-ENL fusion and $t(11 ; 19)$ (q23;p13.3) translocation. 
J. Clin. Oncol, 1999, v.17, p. 191-196.

46. Junien C., Van Heyningen V. Report of the committee on the genetic constitution of chromosome 11. Cytogenet. Cell Genet, 1990; 55: 153-169.

47. Thieblemont C., Pack S., Sakai A., Beaty M., Pak E., Vortmeyer A.O., Wellmann A., Zhuang Z., Jaffe E.S., Raffeld M. Allelic loss of 11 q13 as delected by MEN1-FISH is not associated with mutation of the MEN1 gene in Iymphoid neoplasms. Leukemia, 13: (1): 85-91.

48. Yeyati P.L., Shaknovich R., Boterashvili S., Li, J., Ball H.J., Waxman S., Nason-Burchenal K., Dmitrovsky E., Zelent, A., Licht J.D. Leukemia translocation protein PLZF inhibits cell growth and expression of cyclin A. Oncogene, 1999; 18: 925-934.

49. Genuardi M., Zollino M., Serra A., Leone G., Mancini R., Mango G., Neri G. Long-term cytogen etic effects of antineoplastic treatment in relation to secondary leukemia. Cancer Genet Cytognet, 1998; 33 (2): 201-211.

50. Kempski H.M., Chesslls J.M., Reevs B.R. Deletion s of chromosome 21 restricted to the leukemic cells of children with Down syndrome and leukemia. Leukemia 1997; 11: 1973-1997.

51. Zipursky A., Brown E., Christensen H., Sutherland., Doyle J. Leukemia and/a myeloproliferative syndrome in neonates with Down syndrome. Sem. Perinatol. 1997; 21 (1): 97-101.

52. Satgé D., Sommelet D., Geneix A., Nishi M., Malet P., Vekemans M. A tumor profile in Down Syndrome. Am. J. Med. Genet, 1998; 78: 207-216.

53. Cavani S., Perfumo C., Argusti A., Pierluigi M., Perroni L., Schmiegelow K., Petersen M.B., Cotter F.E., Strigini P., DagnaBricarelli F., Nizetic D. Citogenetic and molecular study of 32 Down syndrome families: potential leukaemia predisposing role of the most proximal segment of chromosome 21q. Br. J. Haematol. 1998; 103 (1): 213-216.

54. Arruda V.R., Figueiredo M.S., Biologia molecular para o hematologista. Temas de hematologia. Belo Horizonte: Imprensa Universitária, 1997; 187-199.
55. Langabeer S.E., Walker H., Roger J.R., Burwett A.K., Wheatley K., Swirsky D., Goldstone A.H., Linch D.C. Incidence of AML1/ETO fusion transcripts in patients entered into theMRC-AML trials. Br. J. Haematol., 1997; 99: 925-928.

56. Govaerts L., Daenen S., Bong B., Breed A., Jong $B$. Deletion (8) (q22) as the only chromosomal abnormality in a patient with RAEB-t with progression to acute myelocytic leukemia. Cancer Genet. Cytogenet. 1998; 30: 319-321.

57. Sandberg A.A. The chromosomes in human cancer and leukemia. 2. ed. New York: Elsevier, 1990; p. 1315.

58. Bhothman, A.R., Lesho, L.J., Somers, K.D., Schellhammer, P.F., Ladaga, L.E., Marchant, D.J. Cytogenetic analysis of four priimary prostatic cultures. Cancer, Genet. Cytogenet, 1989; 37: 241-248.

59. Tajara H.E., Kawasaki-Oyama R.S., Fett-Conte A.C., Gama N.B., Tajara R., Varella-Garcia M. Anomalias cromossômicasem tumores sólidos; aspectos biológicos e a plicações diagnósticas. Rev. Bras. Patol.Clin., 1992; 28 (1).

60. Herens, C., Brasseur, E., Jamar, M., Vierset, L., Schoenen, I., Koulescher, L. Loss of the $Y$ chromosomein bonemarrow cells: results on 1907 consecutive cases of leukaemia na preleukaemia. Clin. Lab. Haematol., 1999; 21 (1): 17-20.

61. Specchia G., Cuneo A., Liso V., Contino R., Pastore D., Gentile E., Rocchi M., Castoldi G.L. A novel translocation $t(1 ; 7)(p 36 ; q 34)$ in three patients with acute myeloid leukaemia.

Br. J. Haematol, 1999; 105: 208-214.

62. Hamaguchi H., Nagata K., Yamamoto K., Fujikawa I., Kobayashi M., Eguchi M. Br. J. Haematol, 1998; 102: 12249-1256.

63. Hatta Y., Yamada Y., Tomonaga M., Miyoshi I., Said J.W., Koeffler H.P. Detailed deletin mapping of the long arm of chromosome 6 in adult T-cell leukemia. Blood, 1999; 93: 613-616.

64. Shapira M.Y., Hirshberg B., Amir G., Rund D. 6;9 translocation in myelodysplastic syndrome. Cancer, Genet. Citogenet, 1999; 112: 57-59.

65. Shinagawa Shinagawa A., Komatsu T., Ninomiya H. Complex translocation $(6 ; 21 ; 8)$, variant of 
$\mathrm{t}(8 ; 21)$, with trisomy 4 in a patient with acute myelogenous leukemia (M2). Cancer, Genet. Citogenet, 1999; 109: 72-75.

66. Wong K.F., Chan J.K., Sin V.C. T-cell prolymphocytic leukemia with a novel translocation $(6 ; 11)(q 21 ; q 23)$. Cancer, Genet Citogenet, 1999; 111: 149-151

67. Amiel A., Mulchanov I., Elis A., Gaber E., Manor Y., Fejgin M., Lishner M. Deletion of 6q27 in chronic lymphocytic leudemia and multiple myeloma detected by fluorescence in situ hybridization. Cancer, Genet. Citogenet, 1999; 112: 53-56.

68. Johansson B., Billeström R., Kristoffersson U., Akerman, Garwicz S., Ahlgren T., Malm C., Mitelman F. Deletion of chromosomearm $3 p$ in hematologic malignancies. Leukemia, 1997; 11.

69. Arranz E., Martinez B., Richart A., Echezarreta G., Roman A., Rivas C.,Benitez J., Increase C-MYC oncogene copy number detected with combined modified comparative genomic hybridization and FISH analysis in a Richter syndrome case with complex karyotype. Cancer, Genet. Cytogenet, 1998; 106 (1): 80-83.
70. Balogh E., Swanton S., Kiss C., Jakab Z.S., Secker-Walker L.M., Oláh É. Fluorescence in situ Hybridization reveals trisomy $2 q$ by insertion into $9 p$ in hepatoblastoma. Cancer, Genet. Cytogenet., 1998; 102: 148-150.

71. Arribas R., Risques R.A., Gonzalez-Garcia I., Masramon L., Aiza G., Ribas M., Capella G., Peinado M.A. Tracking recurrent quantative gen omic alteration in colorectal cancer: allelic losses in chromosome 4 correlate with tumor aggressiveness. Lab.Invest., 1999; 79 (2): 111-122.

72. Gao A.C., Lou W., Ichikawa T., Denmeade S.R. Barrett J.C., Isaacs J.T. Suppression of the tumorigenicity of prostatic cancer cells by gene(s) located on human chromosome 19p13.1-13.2. Prostate, 1999; 38 (1) 46-54.

73. Busson-le Coniat M., Salomon-Nguyen $F$. , Hillion J., Bernard O.A., Berger R. MLL-AF1q fusion resulting from $t(1: 11)$ in acute leukemia. Leukemia, 1999; 13 (2): 302-306.

Recebido: 15/03/1999

Reap resentado: 19/06/2000

Aceito: 15/10/2000 